\title{
Improving the efficiency of image guided brachytherapy in cervical cancer
}

\author{
Sophie Otter, MBBChir, MRCP, FRCR!', Adrian Franklin, MBBS, MRCP, FRCR!', Mazhar Ajaz, PhD, MRCP, FRCR',2, \\ Alexandra Stewart, MD, FRCR, MRCPl,2 \\ ISt Luke's Cancer Centre, Royal Surrey County Hospital, Guildford, University of Surrey, Guildford, United Kingdom
}

\begin{abstract}
Brachytherapy is an essential component of the treatment of locally advanced cervical cancers. It enables the dose to the tumor to be boosted whilst allowing relative sparing of the normal tissues. Traditionally, cervical brachytherapy was prescribed to point A but since the GEC-ESTRO guidelines were published in 2005, there has been a move towards prescribing the dose to a 3D volume. Image guided brachytherapy has been shown to reduce local recurrence, and improve survival and is optimally predicated on magnetic resonance imaging. Radiological studies, patient workflow, operative parameters, and intensive therapy planning can represent a challenge to clinical resources. This article explores the ways, in which 3D conformal brachytherapy can be implemented and draws findings from recent literature and a well-developed hospital practice in order to suggest ways to improve the efficiency and efficacy of a brachytherapy service. Finally, we discuss relatively underexploited translational research opportunities.
\end{abstract}

J Contemp Brachytherapy 20l6; 8, 6: 557-565 DOI: $10.5114 / j c b .2016 .64452$

Key words: brachytherapy, cervical cancer, magnetic resonance imaging.

\section{Purpose}

Brachytherapy is an essential part of the treatment of locally advanced cervical cancer. The ultimate conformal radiotherapy allows a high dose of radiotherapy to be delivered to the tumor whilst relatively sparing the surrounding normal tissue due to the rapid dose fall off with distance from the source. Therefore, high doses can be delivered to the tumor whilst keeping within normal tissue tolerance limits.

Radium was first discovered in 1889 and was initially used for the treatment of skin cancers. Brachytherapy has been used in the treatment of cervical cancer since the 1900s and has been shown to be an essential component of cervical cancer management. Data from the US Patterns of Care Study in 1973 and 1978 showed that the combined use of intracavitary brachytherapy and external beam radiotherapy (EBRT) lead to a 4 year in-field failure rate of $17 \%$ compared to $47 \%$ without brachytherapy $(p<0.001)$, and a 4 year survival of $70 \%$ compared to $37 \%(p<0.001)$ for all stages of disease [1]. Similarly, Montana et al. showed an improvement in disease free survival (DFS) at 2 years from $36 \%$ for EBRT alone to $61 \%$ with the addition of brachytherapy and a reduction in failure rates within the volume of irradiated tissue from $60 \%$ to $48 \%$ in stage III disease [2].

The Manchester dosimetry system was developed in the 1930s [3] and was based on prescription to 'point $A^{\prime}$,
$2 \mathrm{~cm}$ lateral to the center of the uterine canal and $2 \mathrm{~cm} \mathrm{su}-$ perior to the mucous membrane of the lateral fornix along the plane of the uterine canal. The dose at point A was thought to be representative of the minimum dose to most of the malignant tissue. The International Commission on Radiation Units and Measurements (ICRU) Report 38 [4] was published in 1985 and encouraged the use of target volume for dose prescription rather than point $\mathrm{A}$. It also specified the bladder and rectal reference points, which predicted dose to these structures.

With advancements in radiotherapy techniques, such as intensity modulated radiotherapy (IMRT) and stereotactic body radiotherapy (SBRT), there has been a decline in the use of brachytherapy for cervix cancer in the USA from $83 \%$ in 1988 to $58 \%$ in 2009 [5]. However, brachytherapy is independently associated with an improved cancer specific survival and overall survival, and clinical outcome evidence for alternative methods is deeply lacking in comparison [6]. Planning studies have shown that IMRT is not able to achieve target volume doses as high as image-guided brachytherapy when dose constraints $\left(D_{1 c c}\right.$ and $\left.D_{2 c c}\right)$ to the bladder, sigmoid, and rectum are adhered to [7].

Advancements in cervical brachytherapy have included the switch to image guided brachytherapy (IGBT) with the use of computed tomography $(\mathrm{CT})$ or ideally magnetic resonance imaging (MRI). The GEC-ESTRO (Groupe Européen de Curiethérapie [GEC] and European Society
Address for correspondence: Sophie Otter, MBBChir, MRCP, FRCR, Oncology Department, St Luke's Cancer Centre, Royal Surrey County Hospital, Egerton Road, Guildford, GU2 7XX, UK, phone: +44 7970 157925, 凶e-mail: sophie.otter1@nhs.net
Received: 21.09 .2016

Accepted: 16.11 .2016

Published: 30.12 .2016 
for Radiotherapy \& Oncology [ESTRO]) guidelines were published in 2005 to aid with the implementation of IGBT $[8,9]$. This was a move away from prescribing to point $\mathrm{A}$ and instead prescribing the dose to an 'at-risk' volume (predominantly the high risk clinical target volume [HRCTV]). The evaluation of dose to organs at risk (OAR) has also shifted away from the ICRU 38 reference points to a dose volume histogram (DVH) based approach [10]. This allows brachytherapy plans to more accurately define where dose will be rather than predicting where it may be. ICRU 89 further defines and formalizes the principles of the GEC ESTRO guidelines [11].

Retrospective comparison of IGBT and conventional brachytherapy (CBT) at single institutions has shown that IGBT results in a reduction in local recurrence and this subsequently has a beneficial impact on survival $[12,13]$ and toxicity. Therefore, image guided brachytherapy should ideally be the standard of care at every institution.

However, IGBT is a more time-consuming process requiring individualized contouring and planning. It also requires additional resources such as real-time MRI/CT scans, MRI/CT compatible applicators, and availability of 3D treatment planning systems [14]. The additional costs of setting up IGBT are about $10-15 \%$ more than the normal costs of the conventional brachytherapy procedure [15].

The majority of patients will have an intact uterus and therefore will require the placement of an intra-uterine brachytherapy applicator to deliver the correct dose. The insertion of equipment and delivery of an optimized plan require a multidisciplinary team of anesthetists, clinical oncologists, radiographers, nurses, and physicists. This article aims to review the literature and examine techniques, which improve the efficiency of the brachytherapy process within a radiotherapy department, and thus facilitate the introduction of IGBT.

\section{Our institution}

At our institution (St Luke's Cancer Centre, Royal Surrey County Hospital, UK), our patients are treated with IMRT (50.4 Gy in 28 fractions over 5.5 weeks) with concomitant weekly cisplatin chemotherapy. They have daily cone beam CT imaging, which is reviewed online to ensure adequate target coverage. Image guided brachytherapy (up to 21 Gy in 3 fractions) commences after a minimum of 20 fractions of IMRT in weeks 5, 6, and 7. Patients have an MRI the day before brachytherapy, which is reviewed by a gynecologic specialist radiologist. Every IGBT is CT planned at present (see Figure 1). An MRI is performed at fraction 1 with applicator in situ where appropriate and this is fused with CT planning images to aid target definition. Most of our patients receive a spinal anesthetic with intravenous sedation during the insertion procedure. Patients with positive pelvic lymph nodes receive a nodal boost of 5.4-9 Gy in 3-5 fractions.

\section{Anesthesia}

Intra-cavitary brachytherapy (ICBT) is a relatively safe procedure. Major complication rates for low-dose- rate were $<1 \%$ in a retrospective series and most commonly were thromboembolic events (in < $0.3 \%$ ) [16]. On multivariate analysis, older age was the only factor related to perioperative morbidity $(p<0.01)$ [17].

The cervix needs to be dilated to allow the insertion of the intra-uterine tube, which can be a painful procedure, and therefore for patient comfort, we would recommend that patients receive either regional or general anesthetic. It was long assumed that the use of anesthesia allowed more optimal vaginal packing, which in turn would decrease the dose to organs at risk. However, in the preIGBT era, it was shown in a retrospective study using dose to point $A$ and $B$ that dosimetry was not significantly affected whether patients have an anesthetic (spinal or GA) or not [18]. The mean dose to the bladder reference point was not significantly different either but the mean dose to rectal reference point was significantly higher in the anesthetic group (5.09 Gy vs. $4.49 \mathrm{~Gy}, p=0.01$ ). No specific reason was identified for this.

A large retrospective review over 5.5 years was carried out in Vienna of 1622 brachytherapy procedures. $16.8 \%$ of patients had gynecological cancers. The majority of procedures for patients with pelvic malignancies were performed under spinal anesthesia rather than GA (567 vs. 46) [19]. $40 \%$ of patients only required a single dose of local anesthetic through their spinal catheter. A longer duration of procedure tended to require more doses, with a preference to maintain full regional anesthesia until the applicator was removed.

A small series of 34 patients in Japan received a sacral epidural prior to full insertional brachytherapy and self-reported pain on a numeric scale (range 0-10, with $0=$ no pain and $10=$ severe pain). This was compared to patients treated at the same institution without any analgesia and the pain score was significantly lower with the epidural (5.17 vs. $6.80[p=0.035]$ ) [20]. There were no complications associated with the epidural in this series.

In distinction, general anesthetic is associated with significantly more complications than spinal or conscious sedation [21]. A series of 84 fractions of HDR brachytherapy in 18 patients reported 13 complications - 12 in patients having a GA and 1 associated with a paracervical nerve block. Of the fractions delivered under GA, 7 were grade 1 and 5 were grade 2 .

One of the theoretical concerns of regional anesthesia is that it could lead to cervical tumors becoming more hypoxic, and therefore reduce the efficacy of brachytherapy. However, a study of 10 patients showed that there was no significant difference in intra-tumoral $\mathrm{pO}_{2}$ levels before and during spinal anesthesia for cervical brachytherapy [22].

In our department, we favor a spinal anesthetic over general or local anesthetic. This is with a single spinal injection (with no spinal catheter), which maintains adequate anesthesia until applicator removal but allows subsequent discharge later that day. We believe that the data supports this as a comfortable and safe option for the patient both in terms of a low risk of complications and there being no evidence for an adverse effect on tumor radiobiology. 


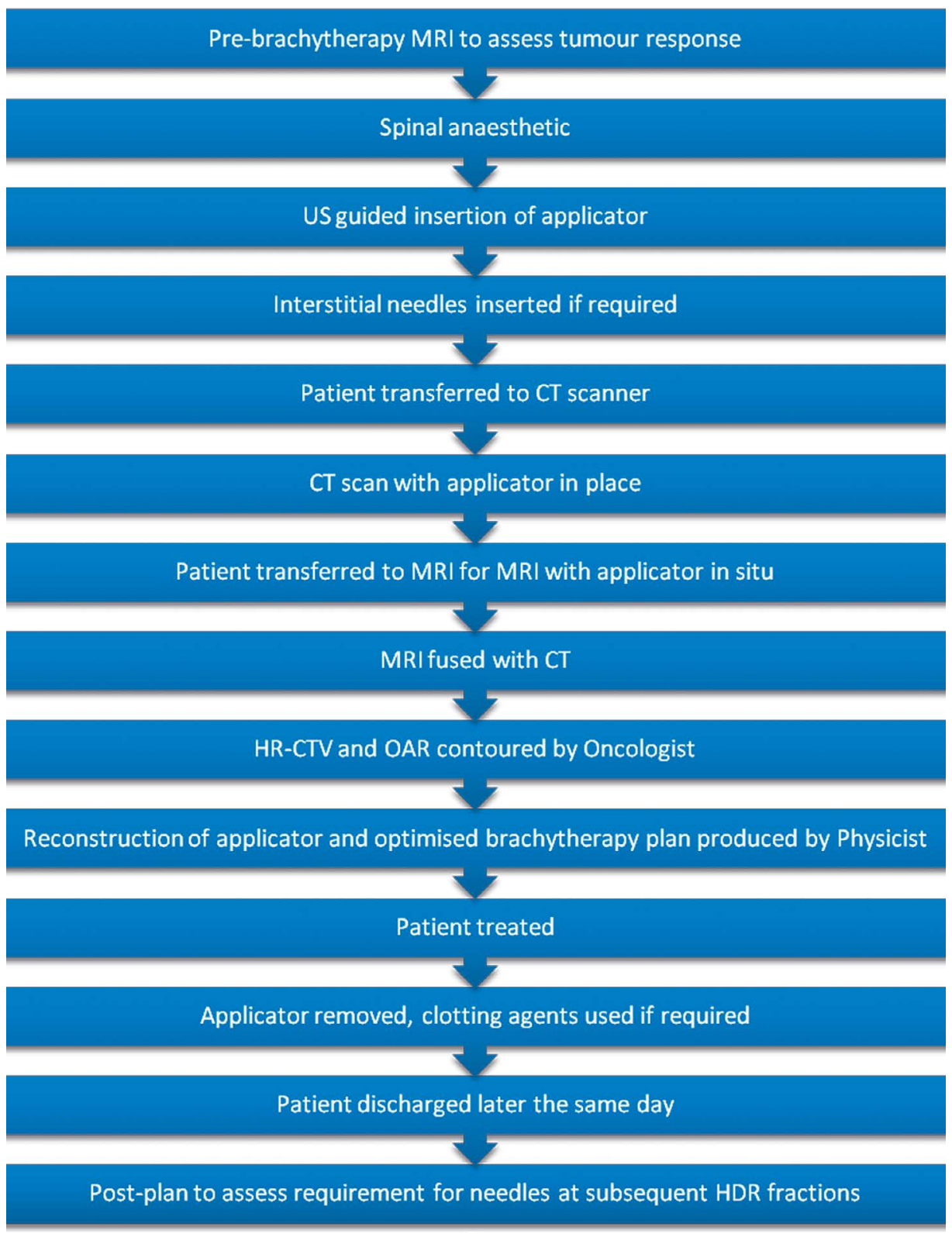

Fig. 1. Flow chart to demonstrate the brachytherapy process at St Luke's Cancer Centre, UK

\section{Accurate applicator placement}

Optimal placement of the tandem and ovoids is essential for an acceptable brachytherapy plan. Patients with implants that were deemed 'ideal' or 'adequate' on the basis of measurements and symmetry on localization films had significantly improved 5-year local control ( $68 \%$ vs. $34 \%, p=0.02$ ) compared to implants deemed 'inadequate' and also had a strong trend toward improved 5 -year survival (60\% vs. $40 \%$ ) [23].

Insertion of the intra-uterine tandem after cervical dilatation can lead to perforation of the uterus. Ultrasound (US) scanning allows direct visualization of the uterine cavity and provides information on the positioning of the uterus. The use of intra-operative ultrasound can minimize the rate of perforation or the need to return to theatre to reposition the equipment to $1.4 \%$ [24]. Davidson et al. [25] reported that using US intra-operatively altered the length and angle of tandem selection in $49 \%$ of cases and reduced the average insertion time from 34 to $26 \mathrm{~min}$ utes $(p=0.01)$. The historical rate of perforation at their institution was $10 \%$ and dropped to $0 \%$.

Certainly, in the pre-IGBT planning era of brachytherapy, intra-operative US was essential particularly in patients with difficult anatomy such as retroverted uterus, previous perforation, or cervical stenosis where assessment on orthogonal films was not sensitive enough to pick up all perforations [26]. However, we feel that the use of US in the IGBT era is still critical as it allows direct visualization of the tandem position within the uterus, and therefore identifies problems with position prior to the CT. This prevents the need to return to theatre to resite the tandem, which is time-con- 
suming and problematic particularly when interstitial needles have been used.

\section{Interstitial needles}

For patients with bulky disease or parametrial invasion, it can be difficult to deliver adequate dose to the HR-CTV whilst achieving OAR toxicity constraints with the tandem and ovoids or ring alone. Various methods can be used for siting the needles such as perineal templates (e.g. Martinez Universal Perineal Interstitial Template and Syed-Neblett [Varian Medical Systems, Inc., Palo Alto, CA, USA]), a tandem and modified ring with holes for the needles (the Vienna applicator-ref), or using tracts within the ovoids as guides (the Utrecht applicator, Nucletron, an Elekta company, Elekta AB, Stockholm, Sweden [27]).

For this reason, the use of interstitial needles is becoming more common. It has been shown that interstitial needles can, in carefully selected patients, lead to increases in $D_{90}$ (dose received by $90 \%$ of the target volume), and $\mathrm{D}_{100}$ for the HR-CTV and reductions in $\mathrm{D}_{2 \mathrm{cc}}$ (the minimum dose received by the most irradiated $2 \mathrm{~cm}^{3}$ ) for sigmoid and bowel compared to an optimized intracavitary implant without interstitial needles [28]. In this series, interstitial needles were used in $41 \%$ of patients and increased the procedure time by an average of 16 minutes. Similarly, the Vienna group have reported the use of interstitial needles in $44 \%$ of patients [12].

Dimopoulos et al. estimated the use of interstitial needles with the Vienna applicator increased procedure time by $15-30$ minutes and planning time by $20-40$ minutes [29]. $13.5 \%$ of needles were placed adjacent to OAR (65\% adjacent to sigmoid and 35\% adjacent to bladder). There was no bladder or sigmoid perforation and these needles could still be used, however, source positions close to the tip were avoided. In this series of 22 patients, the mean number of needles used per application was 3.5.

Retro-EMBRACE is a retrospective multi-institutional study of patients treated with IGBT before EMBRACE was opened (a prospective study of MRI-based IGBT for cervical cancer). Patients treated with intracavitary and interstitial brachytherapy (IC/IS) had a 5\% improvement in 5-year local control rate $(p=0.06)$ compared to intracavitary brachytherapy (IC) alone [30]. In a subgroup analysis of patients with large tumors $\left(\geq 30 \mathrm{~cm}^{3}\right)$, there was a $7 \%$ improvement in 5 -year local control $(p=0.02)$. The improvement in local control is likely to reflect a dose response effect as the IC/IS group had a HR-CTV $D_{90}$ of 92 Gy compared to $83 \mathrm{~Gy}$ in the IC group (i.e. a difference of $9 \mathrm{~Gy}, p \leq 0.01$ ). There was no significant difference in late bladder or GI toxicity. In centers that routinely use IC/IS, the rate of interstitial needle use was $47 \%$.

Mohamed et al. [31] studied 23 patients who had residual parametrial involvement at the time of brachytherapy and therefore had IC/IS brachytherapy. They compared this with ICBT with an EBRT parametrial boost. The mean HR-CTV $D_{90}$ was similar with both techniques but 3 patients would have received a $D_{90}<79$ Gy with the EBRT parametrial boost whereas with IC/IS, all patients had a HR-CTV $D_{90}>84 \mathrm{~Gy} . \mathrm{D}_{2 \mathrm{cc}}$ for OAR were also significantly higher with the EBRT boost. Therefore, the combination of intracavitary and interstitial brachytherapy for patients with parametrial involvement is superior both in terms of higher dose delivered to the HR-CTV and lower dose delivered to the OAR. We would therefore not recommend an EBRT parametrial boost and feel it should be replaced by IGBT.

In our institution, we use the pre-brachytherapy scan to guide subsequent needle placement in patients who still have bulky or asymmetric tumors. Patients with smaller tumors will have the Utrecht applicator placed without interstitial needles. All patients then have a postplan to ascertain whether interstitial needles would have improved $D_{90}$ dose or decreased dose to OAR. The site and depth of needle insertion is then specified for the next insertion. A further post-plan is performed and needle positions altered if required (see Figure 2 and Table 1).

\section{Imaging - MRI/US/CT}

Planning studies have shown that MRI-based IGBT significantly improves target coverage (particularly in larger tumors $>31 \mathrm{cc}$ ) and reduces OAR dose compared to standard planning to point A $[32,33]$.

The Vienna group reported their series of MRIplanned brachytherapy patients (treated from 2001-2003) compared to conventional brachytherapy prescribed to point A (treated from 1998-2000) [12]. They showed an increase in the mean $D_{90}$, which was 81 Gy during the first period and $90 \mathrm{~Gy}$ during the second period ( $p=0.0007)$. Overall survival (OS) at 3 years was increased for tumors $>5 \mathrm{~cm}$ from $28 \%$ in the first period to $58 \%$ in the second period $(p=0.003)$. For tumors $2-5 \mathrm{~cm}$, there was no significant difference. Therefore, the increased dose delivered has greater benefits in bulkier tumors but there will still be benefits of IGBT over conventional brachytherapy (CBT) in smaller tumors such as lower doses to OAR. The grade 3 or 4 late morbidity rate at 3 years was $10 \%$ in the first period and $2 \%$ in the second period.

The Danish group from Aarhus used 99 patients treated in the NOCECA study (EBRT with a simultaneous integrated boost and pulsed-dose-rate BT planned on X-ray imaging) as historical controls, and compared them to their first 5-year experience of IGBT (140 patients from 2005) [34]. The IGBT period used MRI scans with applicators in situ for each implant. Overall survival (OS) was significantly improved with IGBT (63\% to $79 \%$, $p=0.005$ ). It should be noted that concurrent chemotherapy was not given to patients in NOCECA but was given to $79 \%$ of patients treated in the IGBT era, and therefore will account for some of the improvement in OS. However, the use of concomitant chemotherapy has been associated with increased toxicity [35] and yet moderate to severe toxicity was reduced by $50 \%(p=0.02)$ in the IGBT group [34].

The GEC-ESTRO guidelines [8] were devised for MRI based IGBT. However, not all institutions have the resources to provide MRI-based brachytherapy planning for each fraction. Therefore, many institutions use other imaging modalities such as CT and/or US in combination with MRI. In a survey of American Brachytherapy 

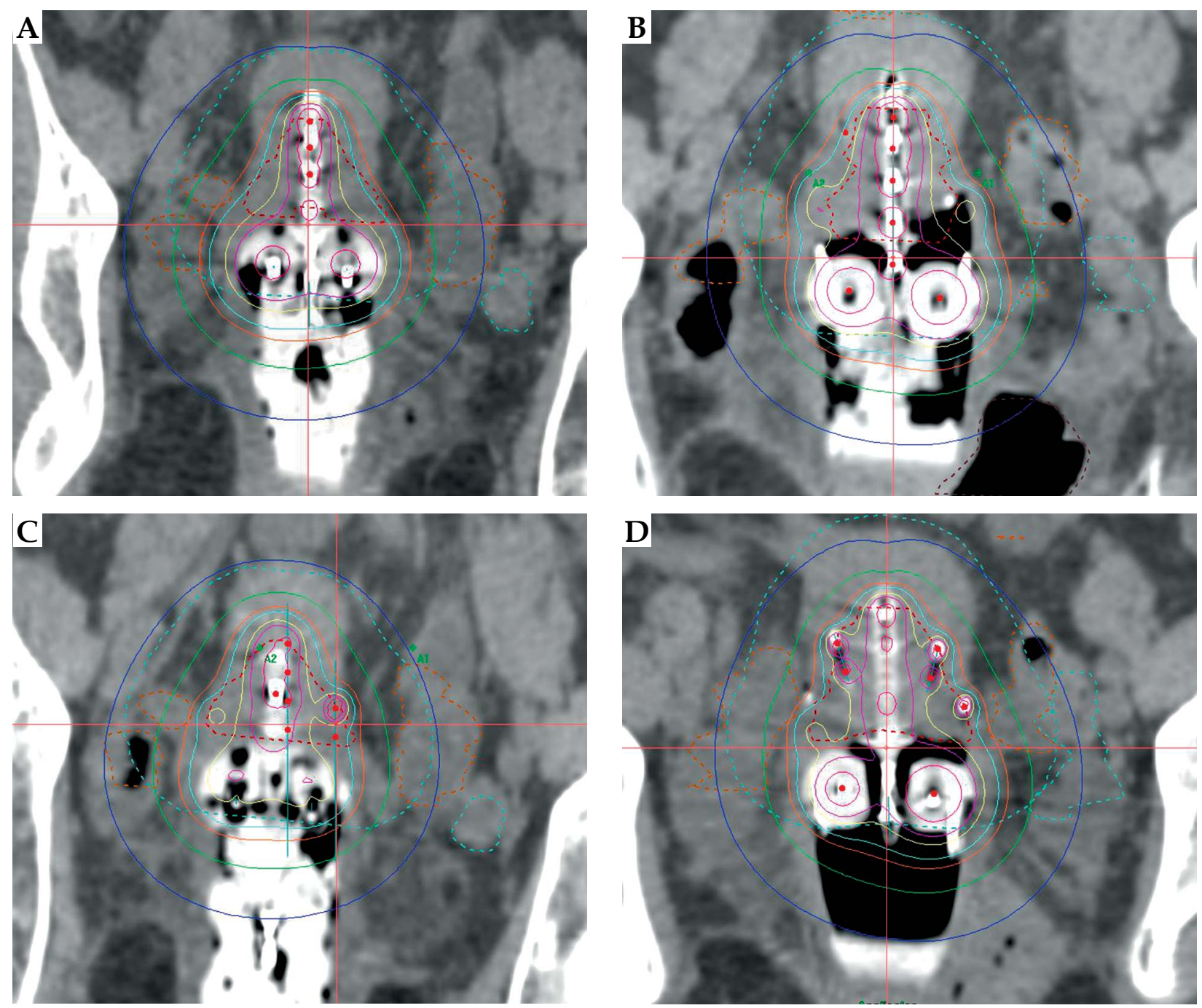

Fig. 2. Brachytherapy plans from a patient with stage IB2 poorly differentiated adenocarcinoma of the cervix. A) Fraction one (no needles). B) Post-plan with 6 needles to improve the coverage of the high risk clinical target volume (HR-CTV) and decrease the dose to the bowel. C) Fraction 2-6 needles sited but only 5 used. D) Fraction 3-6 needles sited and 5 used. The red dotted line denotes the HR-CTV, 100\% - turquoise, 150\% - yellow, 200\% - pink

Society members in $2014,95 \%$ of respondents always use CT and 34\% always use MRI (an increase from $2 \%$ in 2007) [36].

The first report of a hybrid technique using MRI with applicators in situ for the first fraction and CT for subsequent fractions was in 2011. The 2-year local control rate was $88 \%$, DFS $85 \%$, and OS $86 \%$ [37]. Choong et al. [38] have reported results comparing this hybrid technique with IGBT planning based on MRI at each fraction, and showed that the 3-year local control and survival rates were similar. The dosimetry achieved and late toxicity were also similar between the two groups.

Computed tomography-based tumor contours have been shown to over-estimate tumor width [39,40], which can lead to reductions in $\mathrm{D}_{90}$ and $\mathrm{D}_{100}$ [39]. However, Eskander et al. [41] showed that delineation of OAR on both MRI and CT gave similar DVH parameters except for $\mathrm{D}_{2 \mathrm{cc}}$ for bladder, which was higher when outlined on CT than MRI. Therefore, any overestimation of CTV and OAR contours on CT seems to have little clinical significance.
Table 1. The $\mathrm{D}_{90}$ to high risk clinical target volume (HR-CTV) and the $\mathrm{D}_{2 c c}$ values for bladder, bowel, rectum, and sigmoid for the patient depicted in Figure 2. The dose for fraction 3 was reduced to 6.5 Gy to keep the cumulative bowel dose within tolerance

\begin{tabular}{lccc} 
& $\begin{array}{c}\text { Fraction 1 } \\
\text { (Gy) }\end{array}$ & $\begin{array}{c}\text { Fraction 2 } \\
\text { (Gy) }\end{array}$ & $\begin{array}{c}\text { Fraction 3 } \\
\text { (Gy) }\end{array}$ \\
\hline Prescribed dose & 7 & 7 & 6.5 \\
\hline$D_{90}$ to HR-CTV & 7.9 & 8.6 & 7.3 \\
\hline$D_{2 c c}$ bladder & 3.6 & 2.5 & 3.1 \\
\hline$D_{2 c c}$ bowel & 4.8 & 4.5 & 4.1 \\
\hline$D_{2 c c}$ rectum & 1.6 & 1.6 & 0.9 \\
\hline$D_{2 c c}$ sigmoid & 2.8 & 1.5 & 2.4
\end{tabular}

$D_{90}$ - dose received by $90 \%$ of the target volume, HR-CTV - high risk clinical target volume, $D_{2 c c}$ the minimum dose received by the most irradiated $2 \mathrm{~cm}^{3}$ 
Magnetic resonance imaging with applicators in situ has also been used to pre-plan brachytherapy [42]. Patients had the applicators inserted in the radiology department and had an MRI scan prior to fractions 1 and 4 . The applicators were then removed and the patients returned on a subsequent day for re-insertion and treatment. When compared to conventional planning to point A, pre-planning on MRI increased the target dose (5.3 Gy compared to $4.5 \mathrm{~Gy}$ ) and significantly reduced bladder and rectal maximum doses. However, the disadvantage is that patients require more insertional procedures and the reproducibility of inserted applicators needs to be validated. Alternatively, if resources are limited, a standard pre-brachytherapy MRI (without applicators in situ) can be obtained to evaluate tumor shrinkage and to increase the accuracy of HR-CTV contouring on the CT with applicator in situ [43].

An alternative method is US planning, which is used in Melbourne [44]. Ultrasound and MRI are performed at the first fraction with applicator in situ to ensure the differences in measurements of the cervix and uterus are within acceptable limits [45]. For subsequent fractions, US was used to reproduce the position of the tandem in the uterus and the uterus in the pelvis. 5-year local control was $87.5 \%$, which is comparable to results achieved in MRI-based IGBT studies [12,34]. Transcervical US planning has also been shown to be feasible and accurate, based on comparison with a post-insertion MRI [46]. Ultrasound is potentially more cost effective and therefore may be a more feasible option in less developed countries where incidence of cervical cancer is greater.

For these reasons, whilst MRI scanning with applicators in situ for each fraction of IGBT is the gold standard, hybrid techniques with CT or US can give similar results in terms of survival outcome and toxicity rates.

\section{Transfer of patient}

Ideally, imaging would be available in the room either with CT or MRI, but in practice, this is rarely possible. There is therefore a risk of displacement of the tandem, ovoids, and any interstitial needles that have been used. The risk of displacement should be reduced by adequate packing. However, patient transport systems like the Zephyr HDR Patient Positioning and Transfer System (DIACOR, Salt Lake City, Utah, USA) allow the patient to be transported from treatment couch to imaging facility with minimal disruption to the patient, staff, or equipment [47].

\section{Low-dose-rate vs. high-dose-rate}

Low-dose-rate (LDR) and pulsed-dose-rate (PDR) brachytherapy require hospitalization of patients and isolation in a purpose built room. The patient is therefore immobile with an applicator in situ for at least 24 hours (and up to 60 hours in some cases). There is, therefore, the concern of movement of the tandem and ovoids. Highdose-rate brachytherapy, however, is given over approximately 15-30 minutes depending on the source activity and therefore can be given in an outpatient setting. This has an economic benefit and is also more convenient for the patient to be up and mobile more quickly and discharged home the same day. One HDR machine can treat numerous patients in a day, whereas a PDR machine is usually only used for one patient at a time and LDR sources (increasing less commonly used) can only treat one patient at a time.

At our institution, we have a dedicated HDR brachytherapy operating suite with an adjacent recovery bay where patients are cared for whilst their brachytherapy plan is being optimized. This allows us to take the second patient into the brachytherapy suite for insertion of the implant while the first patient is moved to the recovery suite. Therefore, we are able to treat multiple patients in one operating session. Once the second patient has had their CT and satisfactory applicator position is confirmed, the first patient can receive their treatment. The patients have their surgery on mobile operating tables, so each patient remains on their own operating table throughout the process, minimizing transfers.

A meta-analysis of almost 19000 patients included in 15 studies of LDR vs. HDR brachytherapy showed that there was no significant difference in 5-year overall or disease free survival, local recurrence, or bladder and bowel complication rates [48]. These findings have been confirmed with a Cochrane Systematic review, which also showed no difference in terms of survival but there was a slightly increased risk of small bowel complications with HDR compared to LDR [49] in the pre-IGBT era.

\section{Clotting agents}

During the insertion of the tandem, there is the potential to cause bleeding, especially if the tumor is very vascular. At our institution, we use hemostatic agents following the majority of interstitial needle removals to stop bleeding, and therefore allowing patients to be discharged more quickly and to maintain hemoglobin levels during radiotherapy. Tranexamic acid is given intravenously to patients routinely during the procedure whereas floseal and fibrillar are used per vagina if required. Floseal is a liquid hemostatic matrix and has been shown in a systematic review of its use in surgery to reduce the time to achieve hemostasis and the length of hospital stay in a wide array of surgical procedures $(11 \%$ of which were gynecologic) [50].

\section{Planning}

With IGBT for cervical cancer, planning is done in real-time whilst the patient has the applicators in situ. It is important to perform contouring and planning in a time efficient manner. Using the same plan for all fractions of brachytherapy, led to an increase in dose to the bladder and rectum in one study [51]. However, the Danish group found no significant difference in mean $\mathrm{D}_{2 \mathrm{cc}}$ to bladder, rectum, sigmoid, and bowel between a single plan for 2 fractions of IGBT and optimized planning for both fractions [52]. However, for IC/IS brachytherapy, the same applicator geometry was only present in $4 / 33$ patients, 
and therefore for the majority of IC/IS patients, a single plan is not feasible.

At our institution, we contour on a separate platform, thus allowing applicator reconstruction to occur simultaneously. We also use an applicator library rather than reconstructing the applicator every time to maximize time efficiency and improve accuracy. Performing some of the tasks required for the treatment planning procedure in a parallel manner with contouring has been shown to reduce planning time by 25 minutes [53].

\section{Patient experience}

Kirchheiner et al. [54] reported that 30\% of patients experienced symptoms of acute stress disorder one week after treatment and $41 \%$ experience post-traumatic stress disorder 3 months after treatment. Stressful factors identified were pain, organizational problems during brachytherapy, and immobility. Positive factors were support of the treatment team and psychological care, highlighting the importance of communication, and a clinical nurse specialist role for these patients.

\section{Morbidity and mortality}

Petereit et al. [55] reported 30-day morbidity and mortality rates of $5.5 \%$ and $1.6 \%$ in cervical cancer patients having full insertional brachytherapy. In univariate analysis, age was the most predictive factor. American Society of Anesthesiologists (ASA) score, Karnofsky Performance Status (KPS), medical history, and mean procedure time exceeding 160 minutes were also significant factors.

\section{Translational research opportunities}

While technical developments accrue in cervical brachytherapy and undergo more widespread adoption, there is likely to be a limit of achievable benefit in terms of optimal dose distribution. A proportion of tumors will display intrinsic biological radioresistance even to brachytherapy. Importantly, cervical brachytherapy offers direct access to the tumor and the possibility to obtain repeated tissue samples, and thus a high-resolution description of therapy response over time. This is a relatively underexploited facility but with new insights into the tumor microenvironment $[56,57,58]$, particularly immune and inflammatory characteristics, and new therapeutic avenues such as immune checkpoint inhibition (e.g. PD-1 $[59,60,61]$ and CTLA-4 $[62,63])$, there is the opportunity to explore rational new drug-radiation combinations in partnership with technically optimized brachytherapy. This is the focus of a research programme at our institution.

\section{Conclusions}

Image guided brachytherapy reduces the risk of local recurrence and improves survival in patients with locally advanced cervical cancer compared to conventional brachytherapy prescribed to point $\mathrm{A}$. It requires the use of additional imaging modalities such as US, CT, and
MRI to delineate the HR-CTV more accurately. However, it can be difficult to implement in departments due to the increased resources, expertise, and time required. We have outlined in this review how it can be performed efficiently as an outpatient procedure under a spinal anesthetic using US for accurate insertion. Computed tomography and MRI can be used in combination for target volume delineation and OAR outlining allowing each fraction to be individually planned and optimized. Direct tumor access at the time of brachytherapy provides an opportunity for translational studies and further therapeutic progress.

\section{Disclosure}

Authors report no conflict of interest.

\section{References}

1. Lanciano RM, Won M, Coia LR et al. Pretreatment and treatment factors associated with improved outcome in squamous cell carcinoma of the uterine cervix: a final report of the 1973 and 1978 patterns of care studies. Int J Radiat Oncol Biol Phys 1991; 20: 667-676.

2. Montana GS, Fowler WC, Varia MA et al. Carcinoma of the cervix, stage III. Results of radiation therapy. Cancer 1986; 57: 148-154.

3. Meredith W. Radium dosage: The Manchester System. Livingstone, Edinburgh 1967.

4. ICRU report 38. Dose and Volume Specification for Reporting Intracavitary Therapy in Gynaecology. International Commission on Radiation Units and Measurements, Bethesda 1985.

5. Han K, Milosevic M, Fyles A et al. Trends in the utilization of brachytherapy in cervical cancer in the United States. Int J Radiat Oncol Biol Phys 2013; 87: 111-119.

6. Gill BS, Lin JF, Krivak TC et al. National Cancer Data Base analysis of radiation therapy consolidation modality for cervical cancer: the impact of new technological advancements. Int J Radiat Oncol Biol Phys 2014; 90: 1083-1090.

7. Georg D, Kirisits C, Hillbrand M et al. Image-guided radiotherapy for cervix cancer: high-tech external beam therapy versus high-tech brachytherapy. Int J Radiat Oncol Biol Phys 2008; 71: 1272-1278.

8. Haie-Meder C, Pötter R, Van Limbergen E et al. Recommendations from Gynaecological (GYN) GEC-ESTRO Working Group (I): concepts and terms in 3D image based 3D treatment planning in cervix cancer brachytherapy with emphasis on MRI assessment of GTV and CTV. Radiother Oncol 2005, 74: 235-245.

9. Pötter R, Haie-Meder C, Van Limbergen E et al. Recommendations from gynaecological (GYN) GEC ESTRO working group (II): concepts and terms in 3D image-based treatment planning in cervix cancer brachytherapy-3D dose volume parameters and aspects of 3D image-based anatomy, radiation physics, radiobiology. Radiother Oncol 2006; 78: 67-77.

10. Georg P, Lang S, Dimopoulos JC et al. Dose-volume histogram parameters and late side effects in magnetic resonance image-guided adaptive cervical cancer brachytherapy. Int J Radiat Oncol Biol Phys 2011; 79: 356-362.

11. ICRU report 89. Prescribing, Recording, and Reporting Brachytherapy for Cancer of the Cervix. International Commission on Radiation Units and Measurements, Bethesda 2013.

12. Pötter R, Georg P, Dimopoulos JC et al. Clinical outcome of protocol based image (MRI) guided adaptive brachytherapy combined with 3D conformal radiotherapy with or without 
chemotherapy in patients with locally advanced cervical cancer. Radiother Oncol 2011; 100: 116-123.

13. Rijkmans EC, Nout RA, Rutten IH et al. Improved survival of patients with cervical cancer treated with image-guided brachytherapy compared with conventional brachytherapy. Gynecol Oncol 2014; 135: 231-238.

14. Owrangi AM, Prisciandaro JI, Soliman A et al. Magnetic resonance imaging-guided brachytherapy for cervical cancer: initiating a program. J Contemp Brachytherapy 2015; 7: 417-422.

15. Pötter R, Lievens $Y$. What kind of equipment do we need in gynaecological brachytherapy? Clinical, technical and economical aspects for standard and new techniques. Radiother Oncol 2005; 75: S1.

16. Jhingran A, Eifel PJ. Perioperative and postoperative complications of intracavitary radiation for FIGO stage I-III carcinoma of the cervix. Int J Radiat Oncol Biol Phys 2000; 46: 1177-1183.

17. Lanciano R, Corn B, Martin E et al. Perioperative morbidity of intracavitary gynecologic brachytherapy. Int J Radiat Oncol Biol Phys 1994; 29: 969-974.

18. Sharma DN, Chaudhari P, Sharma $S$ et al. Comparison of high-dose-rate intracavitary brachytherapy dosimetry with and without anesthesia in patients with cervical carcinoma. J Appl Clin Med Phys 2014; 15: 4670.

19. Benrath J, Kozek-Langenecker S, Hupfl M et al. Anaesthesia for brachytherapy - 51/2 yr of experience in 1622 procedures. Br J Anaesth 2006; 96: 195-200.

20. Isoyama-Shirakawa Y, Nakamura K, Abe M et al. Caudal epidural anesthesia during intracavitary brachytherapy for cervical cancer. J Radiat Res 2015; 56: 583-587.

21. Lim KH, Lu JJ, Wynne CJ et al. A study of complications arising from different methods of anesthesia used in high-doserate brachytherapy for cervical cancer. Am J Clin Oncol 2004; 27: 449-451.

22. Weitmann HD, Gustorff B, Vaupel P et al. Oxygenation status of cervical carcinomas before and during spinal anesthesia for application of brachytherapy. Strahlenther Onkol 2003; 179: 633-640.

23. Corn BW, Hanlon AL, Pajak TF et al. Technically accurate intracavitary insertions improve pelvic control and survival among patients with locally advanced carcinoma of the uterine cervix. Gynecol Oncol 1994; 53: 294-300.

24. Schaner PE, Caudell JJ, De Los Santos JF et al. Intraoperative ultrasound guidance during intracavitary brachytherapy applicator placement in cervical cancer: the University of Alabama at Birmingham experience. Int J Gynecol Cancer 2013; 23: 559-566.

25. Davidson MT, Yuen J, D'Souza DP et al. Optimization of high-dose-rate cervix brachytherapy applicator placement: the benefits of intraoperative ultrasound guidance. Brachytherapy 2008; 7: 248-253.

26. Small W, Jr., Strauss JB, Hwang CS et al. Should uterine tandem applicators ever be placed without ultrasound guidance? No: a brief report and review of the literature. Int J Gynecol Cancer 2011; 21: 941-944.

27. Nomden CN, de Leeuw AA, Moerland MA et al. Clinical use of the Utrecht applicator for combined intracavitary/interstitial brachytherapy treatment in locally advanced cervical cancer. Int J Radiat Oncol Biol Phys 2012; 82: 1424-1430.

28. Fokdal L, Tanderup K, Hokland SB et al. Clinical feasibility of combined intracavitary/interstitial brachytherapy in locally advanced cervical cancer employing MRI with a tandem/ring applicator in situ and virtual preplanning of the interstitial component. Radiother Oncol 2013; 107: 63-68.

29. Dimopoulos JC, Kirisits C, Petric P et al. The Vienna applicator for combined intracavitary and interstitial brachytherapy of cervical cancer: clinical feasibility and preliminary results. Int J Radiat Oncol Biol Phys 2006; 66: 83-90.

30. Fokdal L, Sturdza A, Mazeron R et al. Image guided adaptive brachytherapy with combined intracavitary and interstitial technique improves the therapeutic ratio in locally advanced cervical cancer: Analysis from the retroEMBRACE study. $R a$ diother Oncol 2016; 120: 434-440.

31. Mohamed S, Kallehauge J, Fokdal L et al. Parametrial boosting in locally advanced cervical cancer: combined intracavitary/interstitial brachytherapy vs. intracavitary brachytherapy plus external beam radiotherapy. Brachytherapy 2015; 14: 23-28.

32. Tanderup K, Nielsen SK, Nyvang GB et al. From point A to the sculpted pear: MR image guidance significantly improves tumour dose and sparing of organs at risk in brachytherapy of cervical cancer. Radiother Oncol 2010; 94: 173-180.

33. Jürgenliemk-Schulz IM, Tersteeg RJ, Roesink JM et al. MRI-guided treatment-planning optimisation in intracavitary or combined intracavitary/interstitial PDR brachytherapy using tandem ovoid applicators in locally advanced cervical cancer. Radiother Oncol 2009; 93: 322-330.

34. Lindegaard JC, Fokdal LU, Nielsen SK et al. MRI-guided adaptive radiotherapy in locally advanced cervical cancer from a Nordic perspective. Acta Oncol 2013; 52: 1510-1519.

35. Tan LT, Zahra M. Long-term survival and late toxicity after chemoradiotherapy for cervical cancer - the Addenbrooke's experience. Clin Oncol (R Coll Radiol) 2008; 20: 358-364.

36. Grover S, Harkenrider MM, Cho LP et al. Image Guided Cervical Brachytherapy: 2014 Survey of the American Brachytherapy Society. Int J Radiat Oncol Biol Phys 2016; 94: 598-604.

37. Beriwal S, Kannan N, Kim H et al. Three-dimensional high dose rate intracavitary image-guided brachytherapy for the treatment of cervical cancer using a hybrid magnetic resonance imaging/ computed tomography approach: feasibility and early results. Clin Oncol (R Coll Radiol) 2011; 23: 685-690.

38. Choong ES, Bownes P, Musunuru HB et al. Hybrid (CT/ MRI based) vs. MRI only based image-guided brachytherapy in cervical cancer: Dosimetry comparisons and clinical outcome. Brachytherapy 2016; 15: 40-48.

39. Viswanathan AN, Dimopoulos J, Kirisits C et al. Computed tomography versus magnetic resonance imaging-based contouring in cervical cancer brachytherapy: results of a prospective trial and preliminary guidelines for standardized contours. Int J Radiat Oncol Biol Phys 2007; 68: 491-498.

40. Viswanathan AN, Erickson B, Gaffney DK et al. Comparison and consensus guidelines for delineation of clinical target volume for CT- and MR-based brachytherapy in locally advanced cervical cancer. Int J Radiat Oncol Biol Phys 2014; 90 : 320-328.

41. Eskander RN, Scanderbeg D, Saenz CC et al. Comparison of computed tomography and magnetic resonance imaging in cervical cancer brachytherapy target and normal tissue contouring. Int J Gynecol Cancer 2010; 20: 47-53.

42. Dolezel M, Odrazka K, Vanasek J et al. MRI-based pre-planning in patients with cervical cancer treated with three-dimensional brachytherapy. Br J Radiol 2011; 84: 850-856.

43. Pötter R, Federico M, Sturdza A et al. Value of Magnetic Resonance Imaging Without or With Applicator in Place for Target Definition in Cervix Cancer Brachytherapy. Int J Radiat Oncol Biol Phys 2016; 94: 588-597.

44. Narayan K, van Dyk S, Bernshaw D et al. Ultrasound guided conformal brachytherapy of cervix cancer: survival, patterns of failure, and late complications. J Gynecol Oncol 2014; 25: 206-213.

45. van Dyk S, Kondalsamy-Chennakesavan S, Schneider M et al. Comparison of measurements of the uterus and cervix ob- 
tained by magnetic resonance and transabdominal ultrasound imaging to identify the brachytherapy target in patients with cervix cancer. Int J Radiat Oncol Biol Phys 2014; 88: 860-865.

46. Petric P, Kirisits C. Potential role of TRAns Cervical Endosonography (TRACE) in brachytherapy of cervical cancer: proof of concept. J Contemp Brachytherapy 2016; 8: 215-220.

47. Nag S. ZephyrTM - a novel "air cushion" patient transportation system for transferring brachytherapy patients for imaging and treatment while minimizing risk of applicator displacement. Poster presented at The American Brachythrapy Society Annual General Meeting, 2011.

48. Lee KK, Lee JY, Nam JM et al. High-dose-rate vs. low-doserate intracavitary brachytherapy for carcinoma of the uterine cervix: Systematic review and meta-analysis. Brachytherapy 2015; 14: 449-457.

49. Liu R, Wang X, Tian JH et al. High dose rate versus low dose rate intracavity brachytherapy for locally advanced uterine cervix cancer. Cochrane Database Syst Rev 2014: CD007563.

50. Echave M, Oyagüez I, Casado MA. Use of Floseal ${ }^{\circledR}$, a human gelatine-thrombin matrix sealant, in surgery: a systematic review. BMC Surg 2014; 14: 111.

51. Davidson MT, Yuen J, D'Souza DP et al. Image-guided cervix high-dose-rate brachytherapy treatment planning: does custom computed tomography planning for each insertion provide better conformal avoidance of organs at risk? Brachytherapy 2008; 7: 37-42.

52. Mohamed S, Nielsen SK, Fokdal LU et al. Feasibility of applying a single treatment plan for both fractions in PDR image guided brachytherapy in cervix cancer. Radiother Oncol 2013; 107: 32-38.

53. Damato AL, Lee LJ, Bhagwat MS et al. Redesign of process map to increase efficiency: Reducing procedure time in cervical cancer brachytherapy. Brachytherapy 2015; 14: 471-480.

54. Kirchheiner K, Czajka-Pepl A, Ponocny-Seliger E et al. Posttraumatic stress disorder after high-dose-rate brachytherapy for cervical cancer with 2 fractions in 1 application under spinal/ epidural anesthesia: incidence and risk factors. Int J Radiat Oncol Biol Phys 2014; 89: 260-267.

55. Petereit DG, Sarkaria JN, Chappell RJ. Perioperative morbidity and mortality of high-dose-rate gynecologic brachytherapy. Int J Radiat Oncol Biol Phys 1998; 42: 1025-1031.

56. Stone SC, Rossetti RA, Lima AM et al. HPV associated tumor cells control tumor microenvironment and leukocytosis in experimental models. Immun Inflamm Dis 2014; 2: 63-75.

57. Kobayashi A, Weinberg V, Darragh T et al. Evolving immunosuppressive microenvironment during human cervical carcinogenesis. Mucosal Immunol 2008; 1: 412-420.

58. García-Rocha R, Moreno-Lafont M, Mora-García ML et al. Mesenchymal stromal cells derived from cervical cancer tumors induce TGF-beta1 expression and IL-10 expression and secretion in the cervical cancer cells, resulting in protection from cytotoxic T cell activity. Cytokine 2015; 76: 382-390.

59. Heeren AM, Punt S, Bleeker MC et al. Prognostic effect of different PD-L1 expression patterns in squamous cell carcinoma and adenocarcinoma of the cervix. Mod Pathol 2016; 29: 753-763.

60. Heeren AM, Koster BD, Samuels S et al. High and interrelated rates of PD-L1+CD14+ antigen-presenting cells and regulatory $\mathrm{T}$ cells mark the microenvironment of metastatic lymph nodes from patients with cervical cancer. Cancer Immunol Res 2015; 3: 48-58.

61. Borghaei H, Paz-Ares L, Horn L et al. Nivolumab versus Docetaxel in Advanced Nonsquamous Non-Small-Cell Lung Cancer. N Engl J Med 2015; 373: 1627-1639.

62. Kosmaczewska A, Bocko D, Ciszak L et al. Dysregulated expression of both the costimulatory CD28 and inhibitory
CTLA-4 molecules in PB T cells of advanced cervical cancer patients suggests systemic immunosuppression related to disease progression. Pathol Oncol Res 2012; 18: 479-489.

63. Hodi FS, O'Day SJ, McDermott DF et al. Improved survival with ipilimumab in patients with metastatic melanoma. N Engl J Med 2010; 363: 711-723. 